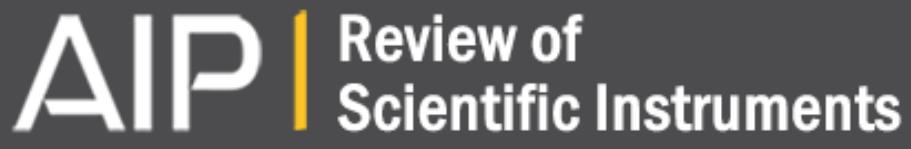

On the use of the double floating probe method to infer the difference between the electron and the heavy particles temperatures in an atmospheric pressure, vortexstabilized nitrogen plasma jet

L. Prevosto, H. Kelly, and B. R. Mancinelli

Citation: Review of Scientific Instruments 85, 053507 (2014); doi: 10.1063/1.4875215

View online: http://dx.doi.org/10.1063/1.4875215

View Table of Contents: http://scitation.aip.org/content/aip/journal/rsi/85/5?ver=pdfcov

Published by the AIP Publishing

\section{Articles you may be interested in}

Characteristics of atmospheric-pressure non-thermal N2 and N2/O2 gas mixture plasma jet

J. Appl. Phys. 115, 033303 (2014); 10.1063/1.4862304

Langmuir probe diagnostics of an atmospheric pressure, vortex-stabilized nitrogen plasma jet J. Appl. Phys. 112, 063302 (2012); 10.1063/1.4752886

Development of a stable dielectric-barrier discharge enhanced laminar plasma jet generated at atmospheric pressure

Appl. Phys. Lett. 100, 253505 (2012); 10.1063/1.4729818

Effects of water addition on $\mathrm{OH}$ radical generation and plasma properties in an atmospheric argon microwave plasma jet

J. Appl. Phys. 110, 053304 (2011); 10.1063/1.3632970

Calculation of the gas temperature in a throughflow atmospheric pressure dielectric barrier discharge torch by spectral line shape analysis

J. Appl. Phys. 103, 063305 (2008); 10.1063/1.2891419

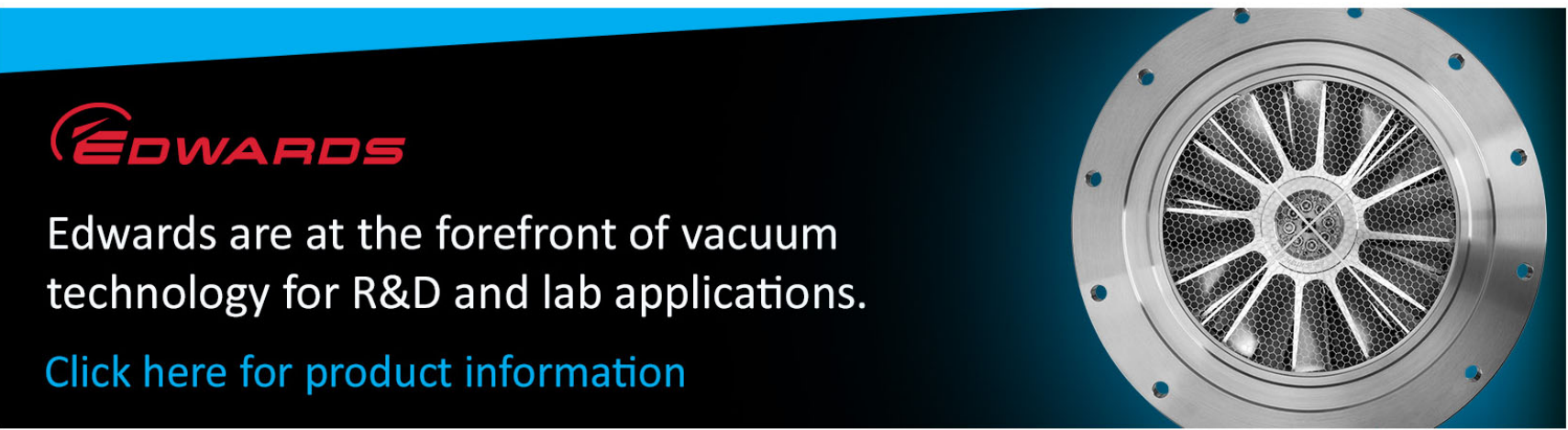




\title{
On the use of the double floating probe method to infer the difference between the electron and the heavy particles temperatures in an atmospheric pressure, vortex-stabilized nitrogen plasma jet
}

\author{
L. Prevosto, ${ }^{1, \text { a) }}$ H. Kelly, ${ }^{1,2}$ and B. R. Mancinelli ${ }^{1}$ \\ ${ }^{1}$ Grupo de Descargas Eléctricas, Departamento Ingeniería Electromecánica, Facultad Regional Venado Tuerto \\ (UTN), Laprida 651, 2600 Venado Tuerto, Santa Fe, Argentina \\ ${ }^{2}$ Instituto de Física del Plasma (CONICET), Departamento de Física, Facultad de Ciencias Exactas y \\ Naturales (UBA) Ciudad Universitaria Pab. I, 1428 Buenos Aires, Argentina
}

(Received 21 March 2014; accepted 25 April 2014; published online 8 May 2014)

\begin{abstract}
Sweeping double probe measurements in an atmospheric pressure direct current vortex-stabilized plasma jet are reported (plasma conditions: 100 A discharge current, $\mathrm{N}_{2}$ gas flow rate of $25 \mathrm{Nl} / \mathrm{min}$, thoriated tungsten rod-type cathode, copper anode with $5 \mathrm{~mm}$ inner diameter). The interpretation of the double probe characteristic was based on a generalization of the standard double floating probe formulae for non-uniform plasmas coupled to a non-equilibrium plasma composition model. Perturbations caused by the current to the probe together with collisional and thermal processes inside the probe perturbed region were taken into account. Radial values of the average electron and heavy particle temperatures as well as the electron density were obtained. The calculation of the temperature values did not require any specific assumption about a temperature relationship between different particle species. An electron temperature of $10900 \pm 900 \mathrm{~K}$, a heavy particle temperature of 9300 $\pm 900 \mathrm{~K}$, and an electron density of about $3.5 \times 10^{22} \mathrm{~m}^{-3}$ were found at the jet centre at $3.5 \mathrm{~mm}$ downstream from the torch exit. Large deviations from kinetic equilibrium were found toward the outer border of the plasma jet. These results showed good agreement with those previously reported by the authors by using a single probe technique. The calculations have shown that this method is particularly useful for studying spraying-type plasma torches operated at power levels of about 15 kW. @ 2014 AIP Publishing LLC. [http://dx.doi.org/10.1063/1.4875215]
\end{abstract}

\section{INTRODUCTION}

Atmospheric pressure thermal plasma jets generated in direct current $(\mathrm{dc})$ non-transferred arc plasma torches are used in a number of applications like plasma processing, surface modifications, spray coatings, material synthesis, and waste treatment. ${ }^{1-5}$ Standard dc non-transferred (sprayingtype) plasma torches operate with a central thoriated tungsten rod-type cathode and a water-cooled annular copper anode. A vortex-type high-pressure flow is injected into the gap between the two electrodes and serves to keep the arc root in a continuous motion over the surface of the anode. Typical torch currents are in the range of a few hundred amperes. The torch voltage depends on the nature of the plasma gas and can vary between 20 or $30 \mathrm{~V}$ for atomic gases up to $100 \mathrm{~V}$ or more when operating with molecular gases. As the gas passes surrounding the arc through the anode-nozzle constriction, it is heated and partially ionized, emerging from the nozzle as a high-velocity non-current carrying plasma jet with mean electron temperatures of about $11000 \mathrm{~K}$. In such torches, large plasma jet fluctuations arise either from ripple in the dc power supply, or random arc root movement at the anode, or combined effects of gas dynamic and electromagnetic instabilities causing cold gas entrainment. ${ }^{2,3,5}$

The Langmuir (electrostatic) probes long ago became one of the most important tool in plasma diagnostics and

a)Electronic mail: prevosto@ waycom.com.ar they are extensively used to obtain spatially and timeresolved information on plasma parameters, mostly in lowpressure plasmas. ${ }^{6-8}$ However these probes have been also successfully employed to study high-pressure highly-ionized plasma discharges, ${ }^{9-18}$ which are typically found in atmospheric pressure, high-current arc torches. Double floating probe method ${ }^{19}$ offers some advantages over (single) Langmuir probes. In particular it is a useful tool for the study of electrode-less plasmas (such as radio frequency plasma discharges ${ }^{6,7,20}$ ) and also in high-pressure, highly ionized plasmas, where the large electron currents drawn by a single Langmuir probe could damage the probe in addition to causing perturbations in the plasma conditions. Another definite advantage of the double probe method over the single probe is its greater independence from the plasma potential fluctuations. ${ }^{6}$ It is known that plasma fluctuations can severely modify the profile of the probe characteristic curve (and its derivatives), leading to substantial errors in the measurements. ${ }^{18,21,22}$ Finally, the double probe method is not sensitive to finite size of a reference electrode, as it is the case in single probe method. ${ }^{23,24}$ Double floating probe method was applied to the study of high-pressure plasma discharges by Rohatgi ${ }^{25}$ and Leveroni and Pfender. ${ }^{26}$ In those works the electron temperature at the periphery of wall-stabilized, one atmosphere, high-current arcs was investigated.

The authors of this work have previously reported Langmuir probe measurements in an atmospheric pressure, vortexstabilized nitrogen plasma jet generated by a spraying-like 
plasma torch. ${ }^{17}$ To derive the electron and heavy particle temperatures, and the plasma composition, it was necessary to take a critical assumption regarding the kinetic deviations with respect to the local thermal equilibrium (LTE). ${ }^{27}$ The work described here concerns the application of the double floating probe method to study such atmospheric pressure plasma jet. A sweeping probe system was employed. To our knowledge, a double floating probe method applied to this type of high-pressure flowing plasma has never been previously considered. A generalization of the standard double floating probe theory for non-uniform plasmas coupled to a non-equilibrium plasma composition model was employed to derive the electron temperature, the heavy particle temperature and the plasma composition of the jet; without appealing to any assumption regarding the kinetic deviations with respect to the LTE.

The paper is organized as follows: the experimental setup is described in Sec. II. The double floating probe theory for non-uniform plasmas is described in Sec. III, while the experimental results and its discussion in terms of the electron and heavy particle temperature, as well as the electron density of the plasma jet, are presented in Sec. IV. The conclusions are summarized in Sec. V.

\section{EXPERIMENTAL SET-UP}

\section{A. Arc plasma torch}

The experiment was carried out using an atmospheric pressure, non-transferred, dc arc torch with a water-cooled thoriated tungsten ( $2 \mathrm{wt} . \%)$ rod-type cathode and a watercooled copper anode-nozzle of $5 \mathrm{~mm}$ inner diameter and $30 \mathrm{~mm}$ in length. The arc was vortex-stabilized and nitrogen was used as the plasma gas. The torch was operated in the so-called restrike mode ${ }^{2,3}$ at a $15 \mathrm{~kW}(150 \mathrm{~V}, 100 \mathrm{~A})$ nomi-

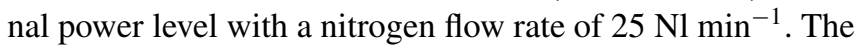
waveform of the cathode (arc) voltage $V_{C}$ (measured with respect to the grounded anode) showing the saw-tooth shape characteristic of the restrike arc mode is given in Fig. 1. More details on the employed arc torch can be found elsewhere. ${ }^{17}$

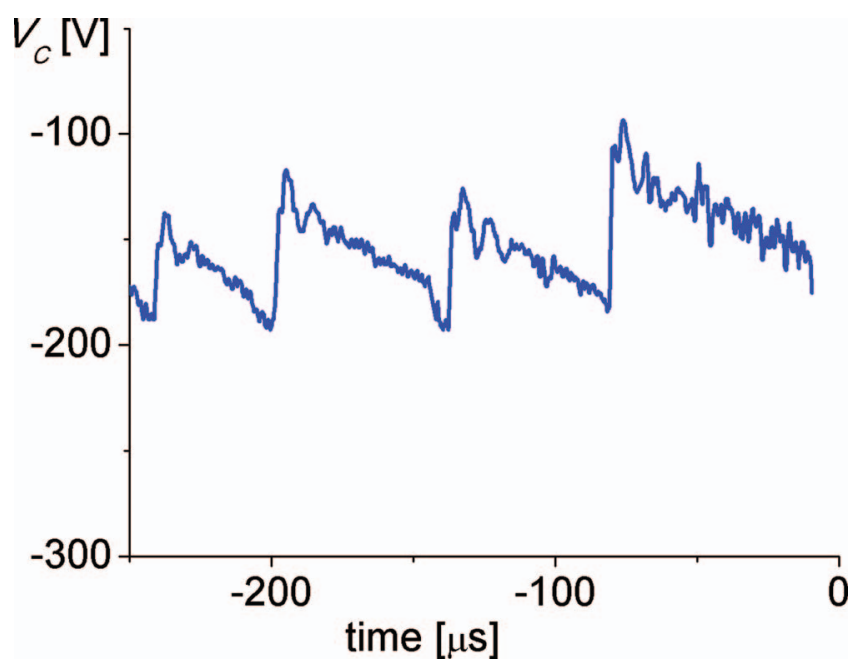

FIG. 1. Cathode voltage waveform corresponding to the torch operating conditions. It can be seen the saw-tooth shape characteristic of the restrike arc mode.

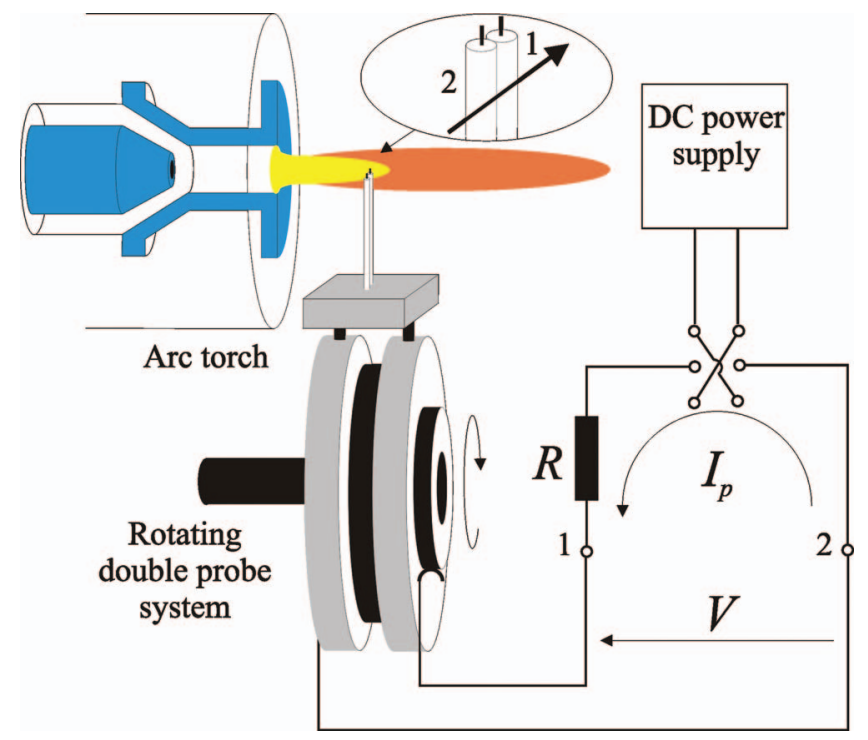

FIG. 2. Schematic of the used experimental set-up, showing the sweeping double floating probe and its biasing circuit.

\section{B. Sweeping double floating probe system}

A schematic of the employed sweeping double floating probe system together with its biasing circuit is showed in Fig. 2. It consisted in a floating rotating conducting holder carrying a double probe mounted in the radial outward direction. The double probe was made of two thin parallel copper wires with a radius $R_{p}=150 \mu \mathrm{m}$, protruding $L_{p}=1.5 \mathrm{~mm}$ from thin capillary glass tubes. The low value of the copper melting temperature (lower than $1500 \mathrm{~K}$ ) rules out the thermoelectric emission from the probe. ${ }^{19}$ The optimal length of the probes with respect to their diameter has been found to be $1.5 \mathrm{~mm}\left(L_{p} / R_{p}=10\right)$ so that the probes only collect ions through the lateral surface. The two wires were $1.5 \mathrm{~mm}$ apart and the probe holder was rapidly swept (probe tip velocity of $22 \mathrm{~m} / \mathrm{s}$ ) through the plasma jet to obtain radial profiles. The probe was aligned perpendicular to the jet with the wires located in an azimuthal plane. The probes length and the rotating holder diameter were chosen large enough in order to consider that the double probe axis was approximately parallel to the line joining the jet axis to the rotating system center during the whole passing of the double probe through the jet. The distance from the torch axis to the rotating center was chosen to ensure that the double probe tip swept the jet cross-section along a diameter. In order to compare the derived plasma quantity profiles with those obtained from a single probe in a previous experiment, ${ }^{17}$ the double probe was located at $3.5 \mathrm{~mm}$ downstream from the torch exit.

The application of a periodically ramping voltage signal (as it is usual for static probes) is not appropriate when performing fast radial sweeps of the probe, because the buildup of the characteristic probe at a given spatial point requires the register of several voltages and corresponding currents at this location. Therefore, a static specific potential value was applied for every sweep of the probe. The voltage was then varied from -31 to $31 \mathrm{~V}$ to obtain the different current values. The probe current $\left(I_{p}\right)$ was calculated by measuring the voltage difference through a resistor $(R=1 \Omega)$ placed in the 
electrical circuit between the two probes, being positive if it flows from the plasma into the probe 2; in accord to the circuit of Fig. 2. The current circulating between the electrically floating probes once immersed in the plasma jet was measured as a function of the applied voltage difference. The probe voltage $(V)$ and the voltage drop across the resistor were simultaneously measured by using a two-channel oscilloscope (Tektronix TDS $1002 \mathrm{~B}$ with a sampling rate of $500 \mathrm{MS} / \mathrm{s}$ and an analogical bandwidth of $60 \mathrm{MHz}$ ). During the experiments there was no correlation between the spatial position of the double probe at a given instant and the corresponding electrical signals value. The time-scale of the plasma fluctuations (mostly caused by the arc restrike mode) determined from the $V_{C}$ waveform showed in Fig. 1 resulted of about $70 \mu \mathrm{s}$. As the probe transit time was in practice similar to the time-scale of the plasma fluctuations, the measured current profiles experienced the natural torch fluctuations. Hence, the current profiles used for the calculations were acquired using the 128 times $(128 \times)$ average acquisition mode of the oscilloscope. Since the probe takes a time of about $5 \mathrm{~s}$ to traverse 128 times the arc, and this time is much larger than the typical fluctuation period, this averaging mode almost quenched the torch fluctuations.

\section{DOUBLE FLOATING PROBE IN HIGH-PRESSURE, HIGHLY IONIZED NON-UNIFORM PLASMAS: FORMULAE}

The connection of probe current measurements with the plasma quantities in high-pressure discharges remains an important, yet incomplete, task. In fact, no comprehensive theory is available for the interpretation of data collected under the plasma conditions contemplated in this study. $6,7,19$

Following Ref. 6, these plasma conditions are classified for electrons as non-local collisionless thin sheath regime (although only weakly ionized plasmas are considered in Ref. 6). This means that $\lambda_{\varepsilon} \gg \delta \gg \lambda_{\mathrm{e}} \gg h$, where $\lambda_{\varepsilon}$ is the relaxation length of the electron energy in elastic collisions with heavy particles, $\delta$ is the characteristic length of the thermal layer around the probe, ${ }^{17} \lambda_{\mathrm{e}}$ is the electron mean-free-path for elastic collisions, and $h$ is the plasma shielding length scale. Under such conditions the electrons from the undisturbed plasma move to the probe in the diffusion regime, conserving their total energy. If the probe operates in the moderately retarding regime (being the probe potential around or more negative than the plasma floating potential) the electron density in the quasi-neutral diffusive layer around the probe follows the Boltzmann distribution with the electric field. The Boltzmann distribution is satisfied since the diffusive electron flux from the plasma to the probe is closely compensated by the electron drift flux from the probe. ${ }^{19}$ As a result, the exponential growth of the voltage-current probe characteristic curve is satisfied at the vicinity of the floating potential and more negative voltages, even in the case of high-pressure (collisional) plasma discharges. A more detailed analysis, ${ }^{28}$ taking into account that the electron flux is strictly nonzero, demonstrated that if the ionization degree of the plasma is not too low, the perturbation created by the probe current is localized only within a near-wall layer, $y_{B}$, adjacent to the probe (in which the electron density deviates from the Boltzmann distribution). If the electron mean-free-path for elastic collisions in a gas of neutral particles, $\lambda_{e a}$, is larger than $y_{B}$, the electron flux to the probe can be evaluated using the electron density at the outer border of that near-wall layer, where they follow the Boltzmann distribution. Therefore, under such plasma conditions the electron current collected by the probe obeys the simple exponential relationship ${ }^{19,23-25,28-31}$

$$
I_{e} \propto N \exp \left(e \frac{V_{p s}}{k T_{e}}\right)
$$

( $N$ is the electron density far from the probe, $e$ is the electron charge, $T_{e}$ is the electron temperature far from the probe, $V_{p s}$ is the probe potential with respect to the plasma potential, and $k$ is the Boltzmann constant).

For ions, the non-local regime ${ }^{6}$ does not exist: $d \gg \lambda_{+}$ $\gg h$ ( $d$ is the diffusion length scale, $\lambda_{+}$is the ion meanfree-path for elastic collisions). The ions from the undisturbed plasma move to the probe in the diffusion regime losing their energy by elastic collisions with a background of cold neutral particles (because the probe cools the adjacent plasma). The value of the ion current collected by the probe is determined by the ambipolar flux from the quasi-neutral region to the outer boundary of the space-charge layer. ${ }^{19}$ Under stationary conditions (the ions transit time across the diffusive layer, $\tau_{d}$, is much shorter than the whole probe transit time through the jet $\tau$ ) and if the plasma in the quasi-neutral region is not fast-moving (the diffusive Péclet number, $P e$, is of the order or less than one) nor in ionization equilibrium (the diffusionionization length, $L$, remains larger than $d$ ), the ion saturation current to the probe is given by the formula (8) of Ref. 17. After some straightforward approximation it can be expressed as

$$
I_{+} \approx S\left(\frac{\pi}{2}\right)^{1 / 2} e N \sqrt{\frac{k T_{e}}{M}}\left(\frac{T_{e}}{T_{h \infty}}\right)^{1 / 2} \frac{\lambda_{+}}{R_{p}}\left(1+\frac{T_{h \infty}}{T_{e}}\right) \frac{1}{\Lambda},
$$

resulting much lower than in collisionless plasmas. The ion mean-free-path in Eq. (2) is defined as $\lambda_{+} \equiv 1 /\left(N+N_{0}\right) Q^{i, 0}$, being $N_{0}$ the neutral particles density (there is no mass flow at the probe wall: the ions diffuse to the probe wall where they recombine and diffuse back into plasma. In such a case $\lambda_{+}$ is inversely proportional to $\left.N+N_{0}\right) \cdot{ }^{32} \Lambda$ is a dimensionless parameter given by

$$
\Lambda \equiv 1.4 T_{h \infty}^{1 / 2}\left(T_{h \infty}+T_{e}\right) \int_{R_{p}}^{R_{p}+d} d r /\left(r\left(T_{e}+T_{h}\right) T_{h}{ }^{1 / 2}\right),
$$

that takes into account the effects of the plasma cooling on the ion current, and varies from a value close to 1 at the jet centre to a value close to 2 at the jet periphery. ${ }^{17}$ The factor 1.4 in Eq. (3) corresponds to an estimation of $1 / \mathrm{ln}\left|1+d / R_{p}\right|$ with $d / R_{p} \approx 1$ ( $S=2 \pi R_{p} L_{p}$ is the probe collecting area, $M$ is the ion mass, and $T_{h \infty}$ is the heavy particles temperature far from the probe).

The formulation also includes the equation of state

$$
\frac{p}{k}=\left(T_{e}+T_{h}\right) N+T_{h} N_{0}
$$


and the generalized Saha equation as derived by Van de Sanden et al., ${ }^{33}$ for the calculation of the non-equilibrium plasma composition

$$
\frac{N^{2}}{N_{0}}=2 \frac{Q_{+}}{Q_{0}}\left(\frac{2 \pi m k T_{e}}{h^{\prime 2}}\right)^{3 / 2} \exp \left(-\frac{E_{I}}{k T_{e}}\right)
$$

( $Q_{+}$and $Q_{0}$ are the statistical weights of atomic ions and atoms, respectively; $h \prime$ is the Planck's constant, $m$ is the electron mass, $E_{I}$ is the first ionization energy of the atoms, and $p$ is the pressure).

The expression describing the current flowing in a double floating probe in non-uniform plasmas was previously derived in Ref. 24,

$$
\frac{I_{e}^{(2)}}{I_{+}^{(2)}}=1-\frac{I_{p}}{I_{+}^{(2)}}=\left(1+\gamma \frac{I_{p}}{I_{+}^{(2)}}\right)^{\vartheta} \exp \left(\frac{e\left(V-V_{f}\right)}{k T_{e, 2}}\right)
$$

$\left(\gamma \equiv \mathrm{I}_{+}{ }^{(2)} / \mathrm{I}_{+}{ }^{(1)}, \vartheta \equiv T_{e, 1} / T_{e, 2}, V_{f}\right.$ is the probe floating potential, superscript " 1 " indicates probe 1 and " 2 " probe 2). This equation represents a theoretical expression for the probe characteristic. All quantities in Eq. (6) are experimentally available. $\gamma$ can be obtained as the absolute value of the ratio of the asymptotic values that the probe current reaches for large negative and large positive $V$, respectively. $T_{\mathrm{e}, 2}$ and $\vartheta$ are obtainable by a two parameter least-square fit of the double probe characteristic curve. For $\gamma=\vartheta=1$ formula (6) reduces to the standard expression, and for $\gamma=0$ it simply becomes similar to the single probe form. ${ }^{19}$

In order to identify typical high-pressure plasma conditions under which the above formulation is justified, plots of the characteristic length ratios $\lambda_{e} / h, \lambda_{+} / h, \lambda_{e a} / y_{B}, L / d, \lambda_{\varepsilon} / \delta$, $\tau / \tau_{d}$, and $P e$, estimated for the unperturbed conditions in a non-equilibrium $\left(\theta \equiv T_{e} / T_{h \infty}=1.5\right)$ one-atmosphere nitrogen plasma, as function of $T_{e}$, are summarized in Fig. 3. It was assumed $R_{p}=150 \mu \mathrm{m}$ and a plasma flow velocity of $100 \mathrm{~m} / \mathrm{s}^{34}$

As it can be seen from Fig. 3, the space-charge layer is collisionless for electrons $\left(\lambda_{\mathrm{e}} \gg h\right)$ in the whole $T_{e}$ range, and also for ions $\left(\lambda_{+} \gg h\right)$ if $T_{e}$ is higher than $8000 \mathrm{~K}$; for $T_{e}$ lower than $11000 \mathrm{~K}$ the ionization process in the adjacent plasma is not perturbed by the probe presence $(L>d)$; for $T_{e}$ higher than about of $8000 \mathrm{~K}$ it results

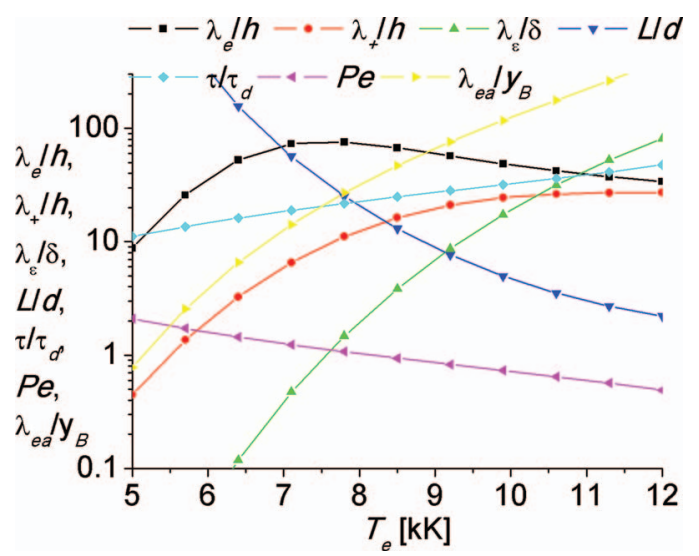

FIG. 3. Characteristic ratios vs. electron temperature for a one-atmosphere non-equilibrium nitrogen plasma. $\left(\lambda_{e a} . \gg y_{B}\right)$ and the "standard" formula given by Eq. (1) applies. Furthermore, for $T_{e}$ higher than around $9000 \mathrm{~K}$ the electron cooling in the plasma region perturbed by the probe can be neglected $\left(\lambda_{\epsilon} . \gg \delta\right)$; the current probe reaches a stationary regime during the transit time in the whole $T_{e}$ range $\left(\tau \gg \tau_{d}\right)$; and the convection effects are not dominant in the probe perturbed region ( $P e \leq 1$, hence $\left.d \approx R_{p}\right)^{28}$ for $T_{e}$ higher than around $9000 \mathrm{~K}$. It is concluded that the above described formulation is justified for $T_{e}$ in the range 9000-11000 K (i.e., ionization degrees in the range $0.01-0.1$ ).

\section{EXPERIMENTAL RESULTS AND DISCUSSION}

\section{A. Double probe characteristics}

In Fig. 4 it is presented the average $(128 \times)$ probe current as a function of the time and corresponding to $V=-31 \mathrm{~V}$. For other negative probe voltages (smaller in absolute value), the $I_{p}$ waveforms retain their shape but with a decreasing amplitude. For positive $V$ values the $I_{p}$ waveforms become negative (with almost the same shape) and with a decreasing amplitude. Under the condition of Fig. 4 probe 2 (negative) is collecting ions which are neutralized by an electron current collected by probe 1 .

To relate this current waveform with the spatial position of the double probe, it must be noted that the maximum of the current waveform (marked in Fig. 4 with the vertical dotted line) will correspond to probe 2 located at the jet centre (where the plasma density is maximum). Accordingly, the minimum $I_{p}$ value obtained for negative $V$ value corresponds to probe 1 located at the jet centre. Therefore, to build an average $I_{p}$ radial profile it must be taking into account the probe separation $(=1.5 \mathrm{~mm})$ and the probes radial speed $(=22 \mathrm{~m} / \mathrm{s})$ to transform the temporal scale into radial scale. This current radial profile is presented in Fig. 5.

It can be seen from Fig. 5 that these profiles appear with a Gaussian-like shape after the quenching of the $N$ fluctuation effects. Nevertheless, some fluctuation level remained present, leading to an experimental uncertainty of about $10 \%$ in $I_{p}$. Since $I_{+}$is almost linearly proportional to $N$ (in Eq. (2) the term $\lambda_{+} / R_{p}$ is only weakly dependent on $N$ ) the average

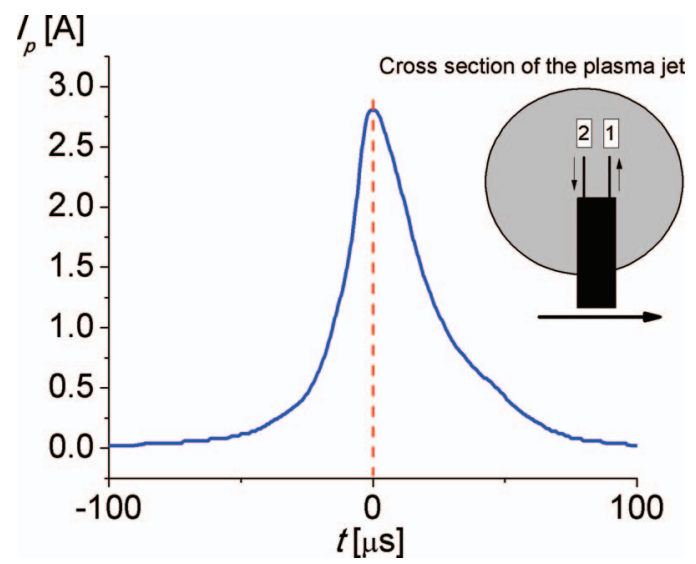

FIG. 4. Average $I_{p}$ waveform for $V=-31 \mathrm{~V}$. The probe location corresponding to the maximum of $I_{p}$ (indicated by the vertical dotted line) is shown in the inset. 


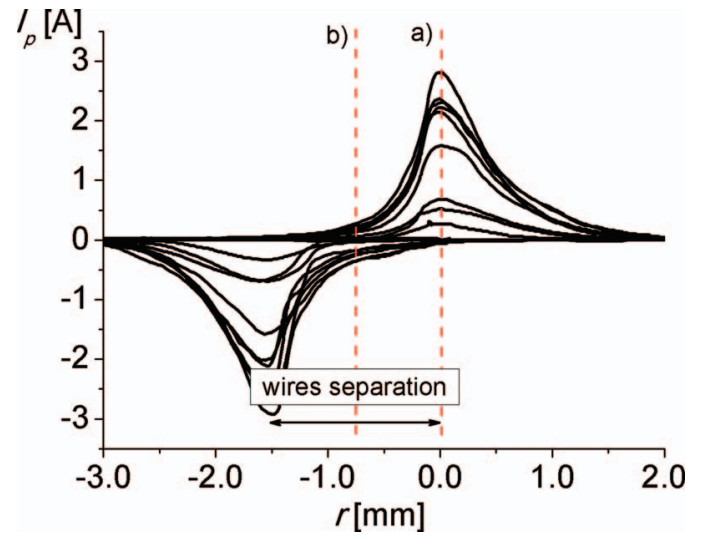

FIG. 5. Average probe current radial profile for different biasing voltages $(-31$ to $31 \mathrm{~V})$. Vertical dotted lines indicate the radial positions selected to calculate the probe characteristic.

values of $I_{p}\left(=I_{+}-I_{e}\right)$ were not significantly affected by fluctuations in $N$.

In Fig. 6 two double probe characteristic curves are presented. They were buildup point by point for given radial distances which are marked by vertical lines in Fig. 5. Fig. 6(a) corresponds to the probe 2 located at the jet centre, while Fig. 6(b) corresponds to the probe 2 located at $0.75 \mathrm{~mm}$ from the jet centre. Note that Fig. 6(a) is presented in a logarithmic
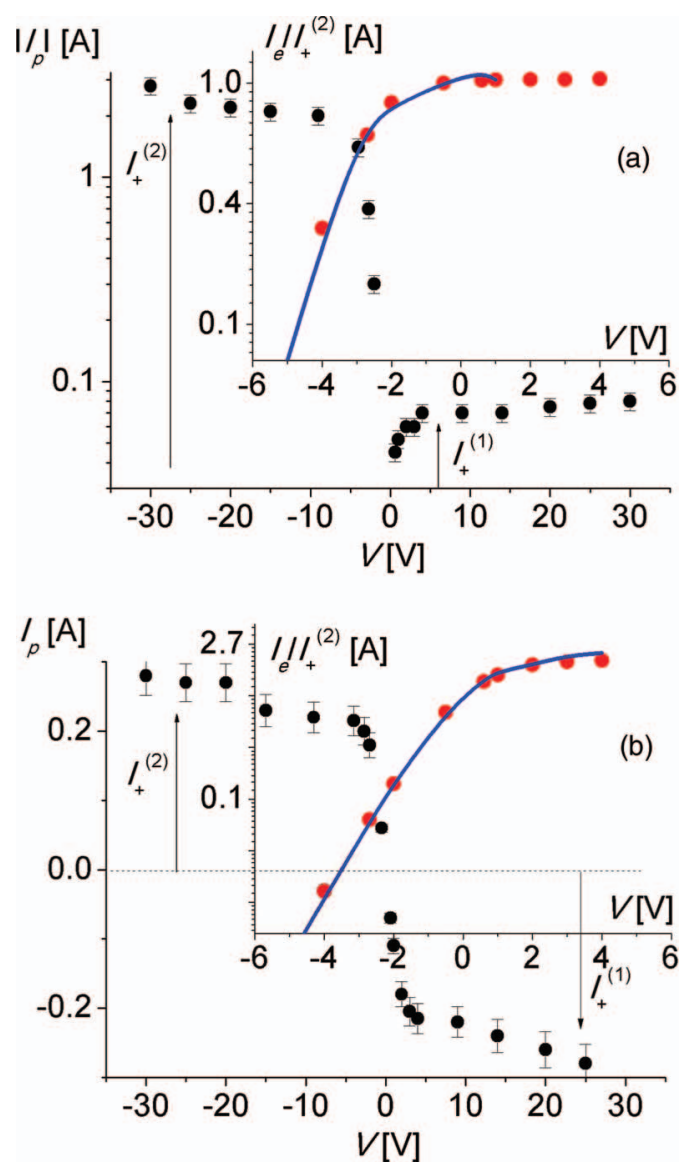

FIG. 6. Double probe characteristic curves: (a) probe 2 located at the jet centre (probe current in absolute value); (b) probe 2 located at $0.75 \mathrm{~mm}$ from the jet axis. The non-linear fit of Eq. (6) to experimental data in each case is showed in an inset. vertical scale using the current modulus due to the large difference between the negative and positive values of the current. The non-linear fits of Eq. (6) to experimental data are showed in the insets of Fig. 6. About 20 measurements were necessary to construct each double probe characteristic curve.

\section{B. Results}

The average electron temperature radial values were obtained from the least square fit of Eq. (6) to the experimental data. The corresponding average heavy particle temperatures and plasma densities were in turn calculated from the measured ion saturation currents by using Eq. (2)-(5); thus avoiding the use of any hypothesis about the non-equilibrium effects on the plasma density. ${ }^{27}$ The $T_{e}$ uncertainty derived from the least square fit of Eq. (6) to the experimental data was within $\pm 3 \%$. Since the statistical and experimental uncertainties involved in the measurements were also of about $\pm 5 \%$, a value of $\pm 8 \%$ can be considered as a realistic estimate of the uncertainty in the electron temperature determination. The resulting $T_{h \infty}$ uncertainty was $\pm 10 \%$, while the uncertainty in $N$ was $\pm 25 \%$. The radial position uncertainty was estimated in $\pm 0.3 \mathrm{~mm}$.

The resulting radial values of the electron and heavy particles temperatures taken at $3.5 \mathrm{~mm}$ downstream from the torch exit (torch operating conditions: 100 A current, $25 \mathrm{Nl} / \mathrm{min} \mathrm{N}_{2}, 1 \mathrm{~atm}$ ) are shown in Figure 7, while Figure 8 gives the obtained plasma density values. For comparison purposes, the corresponding profiles previously derived by the authors with a single probe,${ }^{17}$ under the hypothesis suggested by André et al. ${ }^{27}$ (which relates $T_{e} / T_{h \infty}$ with $N$ ), and for the same dc $\mathrm{N}_{2}$ plasma torch operated at similar conditions, are also shown.

Considering the resulting uncertainties of both temperatures (error bars in Fig. 7), there may be little difference between these two temperatures at the jet axis; but noticeable deviations from kinetic equilibrium appear toward the jet border, with a difference $T_{e}-T_{h \infty}$ in the range 500-3000 K. This is according to Fig. 8, which shows that at the plasma jet centre $N$ reaches a value of around $3.5 \times 10^{22} \mathrm{~m}^{-3}$, which

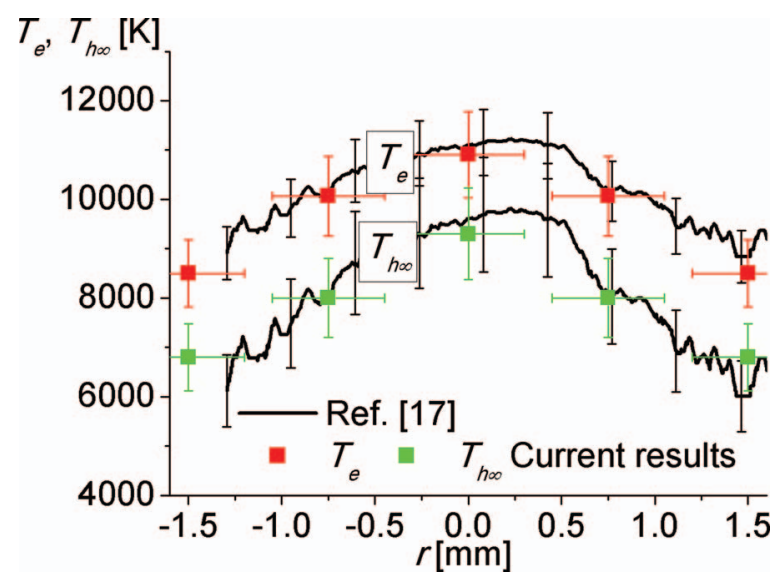

FIG. 7. Resulting radial values of the electron and heavy particles plasma temperatures. The profiles previously derived by the authors by using a single probe (Ref. 17) have been also included. 


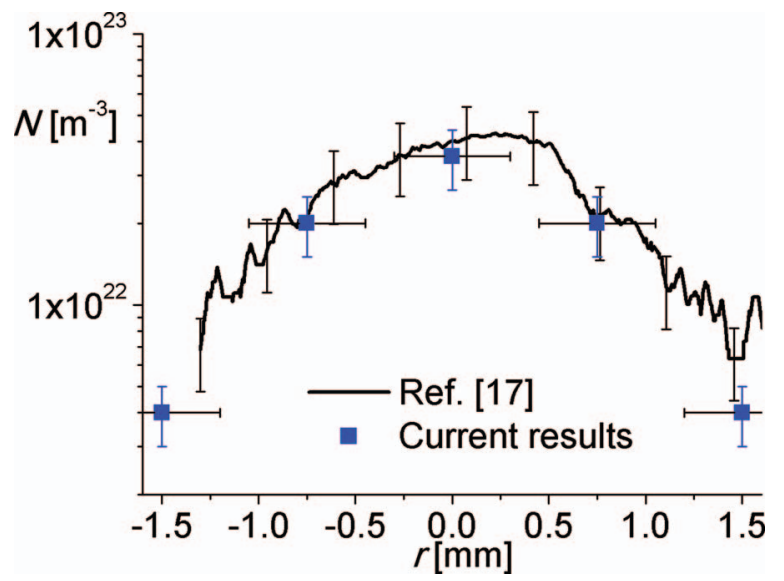

FIG. 8. Resulting values of the plasma density. The density profile previously derived by the authors (Ref. 17) by using a single probe has been also included.

is close to the equilibrium value $\left(\approx 10^{22}-10^{23} \mathrm{~m}^{-3}\right) ;{ }^{1}$ but $N$ decreases sharply as $r$ increases thus causing LTE departures toward the jet border. A global examination of Figs. 7 and 8 shows that the present results are in good agreement with those reported by using a single probe method in the same dc plasma torch, where marked LTE departures were also found. ${ }^{17}$ These results also support the validity of the hypothesis of the kinetic deviations with respect to LTE suggested in Ref. 27.

\section{CONCLUSIONS}

The application of the double floating probe technique to an atmospheric pressure dc vortex-stabilized nitrogen plasma jet has allowed the derivation of the radial values of the electron and heavy particle temperatures; as well as the electron density. The calculation of the temperature values did not require any specific assumption about a temperature relationship between different particle species. The interpretation of the double probe characteristic was based on a generalization of the standard double floating probe formulae for non-uniform plasmas coupled to a non-equilibrium plasma composition model. Large deviations from kinetic equilibrium were found toward the outer border of the plasma jet. These plasma quantities radial values showed good agreement with those previously reported by the authors by using a single probe technique. Several estimations have been shown that this method is particularly useful for studying spraying-type plasma jets operated at power levels of about $15 \mathrm{~kW}$.

\section{ACKNOWLEDGMENTS}

This work was supported by grants from the CONICET (PIP 112/20090/00219) and Universidad Tecnológica Nacional (PID-UTN 1389). H.K. and L.P. are members of the CONICET.

${ }^{1}$ M. Boulos, P. Fauchais, and E. Pfender, Thermal Plasmas, Fundamentals and Applications (Plenum, New York, 1994), vol. 1.

${ }^{2}$ P. Fauchais, J. Phys. D: Appl. Phys. 37, R86 (2004).

${ }^{3}$ P. Fauchais and A. Vardelle, IEEE Trans. Plasma Sci. 25, 1258 (1997).

${ }^{4}$ X. Tu, B. G. Chéron, J. H. Yan, L. Yu, andK. F. Cen, Phys. Plasmas 15, 053504 (2008).

${ }^{5}$ V. Rat and J. F. Coudert, J. Appl. Phys. 108, 043304 (2010).

${ }^{6}$ V. I. Demidov, S. V. Ratynskaia, and K. Rypdal, Rev. Sci. Instrum. 73, 3409 (2002).

${ }^{7}$ V. A. Godyak and V. I. Demidov, J. Phys. D: Appl. Phys. 44, 233001 (2011).

${ }^{8}$ N. St. J. Braithwaite and R. N. Franklin, Plasma Sources Sci. Technol. 18, 014008 (2009).

${ }^{9}$ A. E. F. Gick, M. B. C. Quigley, and P. H. Richards, J. Phys. D: Appl. Phys. 6, 1941 (1973)

${ }^{10}$ C. Fanara and I. M. Richardson, J. Phys. D: Appl. Phys. 34, 2715 (2001).

${ }^{11}$ C. Fanara, IEEE Trans. Plasma Sci. 33, 1072 (2005).

${ }^{12}$ C. Fanara, IEEE Trans. Plasma Sci. 33, 1082 (2005).

${ }^{13}$ L. Prevosto, H. Kelly, and B. Mancinelli, IEEE Trans. Plasma Sci. 36, 263 (2008).

${ }^{14}$ L. Prevosto, H. Kelly, and F. O. Minotti, IEEE Trans. Plasma Sci. 36, 271 (2008).

${ }^{15}$ L. Prevosto, H. Kelly, and B. Mancinelli, IEEE Trans. Plasma Sci. 37, 1092 (2009).

${ }^{16}$ L. Prevosto, H. Kelly, and B. Mancinelli, J. Appl. Phys. 110, 083302 (2011).

${ }^{17}$ L. Prevosto, H. Kelly, and B. R. Mancinelli, J. Appl. Phys. 112, 063302 (2012).

${ }^{18}$ L. Prevosto, H. Kelly, and B. R. Mancinelli, Rev. Sci. Instrum. 84, 123506 (2013).

${ }^{19}$ Y. P. Raizer, Gas Discharge Physics (Springer, Berlin, 1991).

${ }^{20}$ B. M. Annaratonet, G. F. Counsell, H. Kawano, and J. E. Allen, Plasma Sources Sci. Technol. 1, 232 (1992).

${ }^{21}$ A. B. Blagoev, V. I. Demidov, N. B. Kolokolov, and O. G. Toronov, Sov. Phys. Tech. Phys. 26, 1179 (1981).

${ }^{22}$ F. W. Crawford, J. Appl. Phys. 34, 1897 (1962).

${ }^{23}$ S. Saito, M. A. Razzak, S. Takamura, and M. R. Talukder, J. Appl. Phys. 107, 123306 (2010).

${ }^{24}$ J.-S. Chang, J. Phys. D: Appl. Phys. 6, 1674 (1973).

${ }^{25}$ V. K. Rohatgi, Brit. J. Appl. Phys. (J. Phys. D) 1, 485 (1968).

${ }^{26}$ E. Leveroni and E. Pfender, Rev. Sci. Instrum. 60, 3744 (1989).

${ }^{27}$ P. André, J. Aubreton, M. F. Elchinger, P. Fauchais, and A. Lefort, Plasma Chem. Plasma Proc. 21, 83 (2001).

${ }^{28}$ M. S. Benilov, J. Phys. D: Appl. Phys. 33, 1683 (2000).

${ }^{29}$ G. Yang, P. Cronin, J. V. Heberlein, and E. Pfender, J. Phys. D: Appl. Phys. 39, 2764 (2006).

${ }^{30}$ H. E. Porteanu, S. Kühn, and R. Gesche, J. Appl. Phys. 108, 013301 (2010).

${ }^{31}$ A. Shashurin, J. Li, T. Zhuang, M. Keidar, and I. I. Beilis, Phys. Plasmas 18, 073505 (2011).

${ }^{32}$ M. S. Benilov, J. Phys. D: Appl. Phys. 28, 286 (1995).

${ }^{33}$ M. C. M. van de Sanden, P. P. J. M. Schram, A. G. Peeters, J. A. M. van der Mullen, and G. M. W. Kroesen, Phys. Rev. A 40, 5273 (1989).

${ }^{34}$ N. Singh, M. Razafinimanana, and J. Hlinas, J. Phys. D: Appl. Phys. 33, 275 (2000). 